\title{
Feasibility of an Enhanced Recovery Protocol for Elective Pancreatoduodenectomy: A Multicenter International Cohort Study
}

\author{
Didier Roulin $^{1} \cdot$ Emmanuel Melloul $^{1} \cdot$ Björn Erik Wellg $^{2} \cdot$ Jakob Izbicki $^{2}$ • \\ Dionisios Vrochides ${ }^{3} \cdot$ Mustapha Adham ${ }^{4} \cdot$ Martin Hübner $^{1} \cdot$ Nicolas Demartines $^{1}$
}

Published online: 8 April 2020

(C) Société Internationale de Chirurgie 2020

\begin{abstract}
Background The first enhanced recovery after surgery (ERAS) guidelines for pancreatoduodenectomy (PD) were developed in 2012. The study aimed to assess compliance and outcomes of an ERAS protocol for PD, to study correlation between compliance and outcomes, and to identify risk factors for complications.

Methods Retrospective cohort analysis is based on a prospective database, including all consecutive patients undergoing elective PD within an ERAS program in four centers: Lausanne University Hospital (Switzerland), Carolinas Medical Center (United States), Edouard Herriot Hospital (France), and University Medical Center Hamburg-Eppendorf (Germany). Patients' characteristics, postoperative outcome and ERAS compliance were assessed. Logistic regression analysis was performed to assess predictors of postoperative complications.

Results Between October 2012 and June 2017, 404 consecutive patients underwent PD. Median length of stay was 14 days with $11.3 \%$ readmission rate. Mean overall compliance was $62 \%$, with pre-, intra- and postoperative compliance of $93 \%, 80 \%$ and $30 \%$, respectively. Overall compliance $\geq 70 \%$ versus $<70 \%$ was significantly associated with a reduction in complications $(p=0.029)$ and length of stay $(p<0.001)$. Avoidance of postoperative nasogastric tube $(\mathrm{OR}=0.31, p=0.043)$, mobilization on day of surgery $(\mathrm{OR}=0.28, p=0.043)$, and mobilization more than $6 \mathrm{~h}$ on postoperative day $2(\mathrm{OR}=0.45, p=0.001)$ were independent predictors of reduced overall complications.

Conclusions Implementation of enhanced recovery for PD is challenging, especially in the postoperative period. Overall compliance with ERAS protocol $\geq 70 \%$ was associated with decreased complications and length of stay. Specific ERAS elements, such as avoidance of postoperative nasogastric tube and early mobilization, independently improved outcomes.
\end{abstract}

Nicolas Demartines

demartines@chuv.ch

1 Department of Visceral Surgery, Lausanne University Hospital CHUV, University of Lausanne UNIL, Rue du Bugnon 46, 1011 Lausanne, Switzerland

2 Department of General, Visceral and Thoracic Surgery, University Medical Center Hamburg-Eppendorf, Hamburg, Germany
3 Division of HPB Surgery, Carolinas Medical Center, Charlotte, USA

4 Department of Digestive, Hepato-Biliary and Pancreatic Surgery, Edouard Herriot Hospital, HCL, Lyon Sud Faculty of Medicine, UCBL1, Lyon, France 


\section{Introduction}

Enhanced recovery after surgery (ERAS) was initially developed for colorectal surgery in order to reduce the impact of the postsurgical metabolic stress response and improve postoperative outcomes based on the implementation of a multidisciplinary and multimodal pathway. ERAS pathways are composed of multiple individual evidence-based elements which cover the entire patient's journey, from the first referral until the postoperative follow-up [1]. First guidelines for pancreatoduodenectomy (PD) were published in 2012 [2], and the recommendations were mostly based on PD performed outside an ERAS setting or extrapolated from other surgical procedures such as colorectal surgery. Therefore, limited data are currently available on the feasibility and benefits of implementing ERAS pathway according to the existing ERAS guidelines for PD. Moreover, the impact of each individual elements of an ERAS pathway for PD has not been studied yet.

This study aimed to assess the feasibility of an enhanced recovery program for PD in a multicenter setting by analyzing the compliance to each ERAS items. The secondary aim was to analyze the effect of compliance on outcomes, and to identify predictors of complications.
Material and methods

\section{Inclusion}

Retrospective cohort analysis is based on a prospective database, including all consecutive patients undergoing open, laparoscopic or robotic elective PD (classic Whipple or pylorus preserving PD) within an ERAS pathway in four tertiary centers: Lausanne University Hospital (Switzerland), Carolinas Medical Center (United States), Edouard Herriot Hospital (France), and University Medical Center Hamburg-Eppendorf (Germany). Inclusion started from the date of the first patient operated within an implemented ERAS pathway in each center (8th October 2012 at Lausanne University Hospital, 1st September 2015 at Carolinas Medical Center, 17th February 2016 at University Medical Center Hamburg-Eppendorf, and 26th June 2016 at Edouard Herriot Hospital) until 30th June 2017. Patients less than 18 years old, operated in an emergency setting, or without consent were not included. In each center, the surgeon leader (J.I., M. A., D. V. and N. D.) built up a multidisciplinary ERAS team including surgeons, anaesthetists and nurses champions [3].The ERAS pathway in the four centers was based on the perioperative elements as described in Table 1 according to the ERAS recommendations for PD [2].

Table 1 Definitions of enhanced recovery elements used for compliance calculation

\begin{tabular}{|c|c|}
\hline ERAS elements & Compliance definition \\
\hline Preadmission patient education & Dedicated preoperative counseling and education \\
\hline Oral bowel preparation & No bowel preparation \\
\hline Preoperative oral carbohydrate & $\begin{array}{l}\text { Carbohydrate drink until } 2 \mathrm{~h} \text { before surgery, except in case of mechanical obstruction, gastroparesis, or } \\
\text { uncontrolled diabetes }\end{array}$ \\
\hline $\begin{array}{l}\text { No preoperative long-acting sedative } \\
\text { medication }\end{array}$ & No preoperative long-acting sedatives on day of surgery \\
\hline Thrombotic prophylaxis & Preoperative low-molecular-weight heparin \pm sequential compression device \\
\hline Antibiotic prophylaxis & Antibiotic prophylaxis before skin incision \\
\hline PONV prophylaxis & PONV prophylaxis if two or more risk factors (female, non-smoker, previous PONV/motion sickness) \\
\hline Epidural & Thoracic epidural, except when contra-indicated \\
\hline Upper-body heating cover & Use of upper-body forded-air heating cover intraoperatively \\
\hline No postop nasogastric tube & Removal of nasogastric tube before end of surgery \\
\hline Early abdominal drain removal & Early drain removal on POD 3 if low risk \\
\hline Termination urinary drainage POD 2 & Transurethral catheter removal on POD 2 \\
\hline Stimulation of gut motility & Oral laxatives and/or chewing gum given postoperatively \\
\hline Increase weight POD 1 & Increase in weight of less than $2 \mathrm{~kg}$ \\
\hline Balanced iv fluids POD 0 & Less than $3500 \mathrm{ml}$ intravenous fluid on day of surgery \\
\hline Termination iv fluids & Termination of intravenous infusion less than 2 postoperative nights \\
\hline Mobilization on day of surgery & Any mobilization (to walk, to sit on a chair or rising from bed) \\
\hline Mobilization on POD 1 & Mobilization in total for more than $4 \mathrm{~h}$ \\
\hline Mobilization on POD 2 & Mobilization in total for more than $6 \mathrm{~h}$ \\
\hline
\end{tabular}

$P O N V$ postoperative nausea and vomiting, $P O D$ postoperative day 


\section{Data collection}

Data on patients' characteristics, surgery, compliance and outcome were collected prospectively by each center from the preoperative period until postoperative day 30. Data were coded and entered on an online web-based central database, the ERAS Interactive Audit System (www.eras society.org, ENCARE, Krista, Sweden) as previously described [4]. An extraction of the data from the ERAS Interactive Audit System was performed in December 2017 and data from each center were merged in a single database for analysis. Ethical committee approval was individually obtained by each center (Lausanne University Hospital: \# 2016-01815, Carolinas Medical Center: \# 06-12-34E, University Medical Center Hamburg-Eppendorf: \# PV 5779, and Edouard Herriot Hospital: \# 16/09/06). The study was performed and reported according to the STROBE statement [5] and to the Reporting on ERAS Compliance Outcomes, and Elements Research (RECOvER) checklist [6].

\section{Definitions}

Compliance to pre-, intra-, and postoperative elements was assessed as specified in the ERAS society guidelines for PD [2] and reported as compliant, non-compliant, or missing. Overall compliance was calculated as the total of compliant elements divided by all 19 ERAS perioperative elements (Table 1). Primary length of stay was the total number of days in hospital from the operation until discharge. Total length of stay was the total number of days following readmission within 30 postoperative days added to the primary length of stay. Complications were defined as any adverse event occurring in the postoperative period until 30 days after surgery, and were graded according to the Dindo-Clavien classification [7]. Clavien grade I-II were considered as minor, and grade IIIa to IVb as major complication. Pancreas surgery specific complications, such as post-pancreatectomy hemorrhage, delayed gastric emptying or pancreatic fistula were graded A to $\mathrm{C}$ according to the respective International Study Group of Pancreatic Surgery classifications (ISGPS) [8-10].According to the 2016 ISGPS update [11], only clinically relevant pancreatic fistula (Grade B-C) were reported. Patients were also classified according to the American Society of Anesthesiologists (ASA) grade (low, I-II; high III-V) [12], preoperative World Health Organization (WHO) performance status [13] (good, 0; reduced I-III) and body mass index (BMI).

\section{Statistical analysis}

To assess the correlation between compliance and postoperative outcome, complications (overall and major) as well as length of stay (primary and total) were compared between patients with less or more than $70 \%$ of compliance. An overall compliance rate of $70 \%$ or more was defined as cutoff according to previous studies $[14,15]$ The Fischer test for complications and the Mann-Whitney $U$ test for length of stay were used.

Binary logistic regression analysis with overall complication and major complication as dependent variable was performed with following independent variables: patient (age, gender, BMI, ASA grade, preoperative WHO performance score, surgery (procedure, approach, length of surgery) and ERAS specific elements. Only factors with at least $10 \%$ of patients differing from the rest of the cohort (i.e., $n \geq 39$ ) were included in the regression analysis. Multiple logistic regression analysis was further performed including all previous independent variables with a $p$ value $<0.1 . p<0.05$ was considered statistically significant. SPSS version 25 (IBM Corp., Armonk, NY) was used for data analysis.

\section{Results}

A total of 404 patients undergoing elective PD at the four academic centers were prospectively entered into the ERAS Interactive Audit System between 1st October 2012 and 30th June 2017. Fourteen patients refused the use of their data and were excluded. The number of included patients per center was as following: $n=164$ at Lausanne University Hospital; $n=105$ at Carolinas Medical Center; $n=38$ at University Medical Center Hamburg-Eppendorf; and $n=83$ at Edouard Herriot Hospital.

The characteristics of included patients are displayed in Table 2 . The study population was in general elderly with a mean age of 65 years and with more than $70 \%$ of patients with reduced WHO performance status. The classical Whipple was performed in 52\% of the patients, and 93\% of the operations were performed by open surgery. Vascular resection, including venous, arterial and combined, was performed in $21 \%$ of patients $(n=81)$.

\section{Compliance}

The compliance to each ERAS element is described in Fig. 1. Mean overall compliance was $62 \%$, with a compliance in the pre-, intra-, and post- operative period of $93 \%, 80 \%$ and $30 \%$, respectively. No significant difference in compliance between centers was observed. Missing data for compliance items were on average 10\%. Difficult 
Table 2 Baseline characteristics of patients undergoing elective pancreatoduodenectomy within an ERAS pathway $(n=390)$

\begin{tabular}{|c|c|c|}
\hline Category & $N$ & $\%$ \\
\hline Gender, male & 217 & 55.6 \\
\hline Age, mean (SD), years & $65.3(11.6)$ & \\
\hline BMI, mean (SD), $\mathrm{kg} / \mathrm{m}^{2}$ & $25.5(4.7)$ & \\
\hline \multicolumn{3}{|l|}{ ASA } \\
\hline $1-2$ & 186 & 47.7 \\
\hline $3-4$ & 203 & 52.0 \\
\hline Neoadjuvant chemotherapy & 549 & 12.6 \\
\hline \multicolumn{3}{|l|}{ Preoperative WHO performance status } \\
\hline 0 & 112 & 28.7 \\
\hline $1-3$ & 275 & 70.6 \\
\hline \multicolumn{3}{|l|}{ Procedure type } \\
\hline Pancreaticoduodenectomy (PD) & 203 & 52.1 \\
\hline Pylorus-preserving PD & 187 & 47.9 \\
\hline \multicolumn{3}{|l|}{ Surgical approach } \\
\hline Open/converted & 362 & 92.9 \\
\hline Laparoscopic & 6 & 1.5 \\
\hline Robotic & 22 & 5.6 \\
\hline \multicolumn{3}{|l|}{ Vascular resection } \\
\hline Venous & 66 & 16.9 \\
\hline Arterial & 7 & 1.8 \\
\hline Arterial and venous & 8 & 2.1 \\
\hline Missing & 3 & 0.8 \\
\hline \multicolumn{3}{|l|}{ Diagnosis } \\
\hline Primary adenocarcinoma & 297 & 76.2 \\
\hline Other primary malignancy & 34 & 8.7 \\
\hline Metastasis or recurrence of malignant disease & 3 & 0.8 \\
\hline Benign tumor & 25 & 6.4 \\
\hline Chronic pancreatitis & 6 & 1.5 \\
\hline Others & 24 & 6.2 \\
\hline Missing & 1 & 0.3 \\
\hline
\end{tabular}

$S D$ standard deviation, BMI body mass index, WHO World Health Organization, ASA American Society of Anesthesiologists

elements to achieve (with a compliance less than 50\%) were mainly found in the postoperative period. They concerned two main areas: fluid balance and urinary drainage removal (average compliance $=19 \%$ ) as well as adequate postoperative mobilization (average compliance for day 0 , 1 , and $2=40 \%$ ).

\section{Outcomes}

Postoperative outcomes are summarized in Table 3 . Overall, complications within 30 postoperative days occurred in $83.7 \%$ of the patients, with a major complications rate of $36.9 \%$. The 30-day mortality rate was $3.1 \%$.
A compliance with ERAS pathway $\geq 70 \%$ was associated with an improved postoperative outcome as detailed in Table 4. Patients achieving a compliance above $70 \%$ had a significantly reduced primary and total length of stay $(p<0.001)$ and underwent significantly less overall and major complications $(p=0.029$ and $p=0.012$, respectively). The reduction in complications with compliance $\geq 70 \%$ was significant for respiratory $(p=0.022)$ and infectious $(p=0.030)$ complications.

\section{Predictive factors of overall complications}

No patient's or surgical characteristics affected the overall complications rate on multivariable logistic regression analysis. Avoidance of postoperative nasogastric tube (OR $0.31 ; 95 \%$ CI $0.14-0.97 ; p=0.043$ ), mobilization on day 0 of surgery (OR $0.28 ; 95 \%$ CI $0.14-0.97 ; p=0.043$ ) and mobilization more than $6 \mathrm{~h}$ on the second postoperative day (OR $0.45 ; 95 \%$ CI $0.22-0.93 ; p=0.001$ ) were independent predictors of decreased overall complications (Table 5).

\section{Predictive factors of major complications}

Female patients were associated with reduced major complications (OR $0.53 ; 95 \%$ CI $0.33-0.85 ; p=0.009$ ) (Table 6). Altered preoperative WHO performance (more than 0) was an independent predictor of increased major complications (OR 2.43; 95\% CI 1.35-4.36; $p=0.003$ ). Early mobilization on the day of surgery was the only independent predictor among ERAS items and was associated with reduced major complications (OR $0.42 ; 95 \% \mathrm{CI}$ $0.22-0.77 ; p=0.005$ ).

\section{Discussion}

In this international multicenter study including patients undergoing PD without any selection criteria, high compliance to ERAS was obtained only in the pre- and intraoperative period. As expected, a high overall compliance (defined as more than 70\%) was associated with significant reduction in complications and length of stay. Specific postoperative ERAS elements, such as avoidance of nasogastric tube, as well as early mobilization at postoperative day 0 and 1 were independent factors associated with decreased complications.

Postoperative outcomes such as LOS, readmission and clinically relevant pancreatic fistula were in the range of benchmark cutoffs as recently defined for pancreatic surgery [16]. Postoperative complications Grade $\geq 3$ and mortality was $37 \%$, and $3.1 \%$ in the present study, which is above the benchmark cutoffs of $\leq 30 \%$, and $\leq 1.6 \%$, 


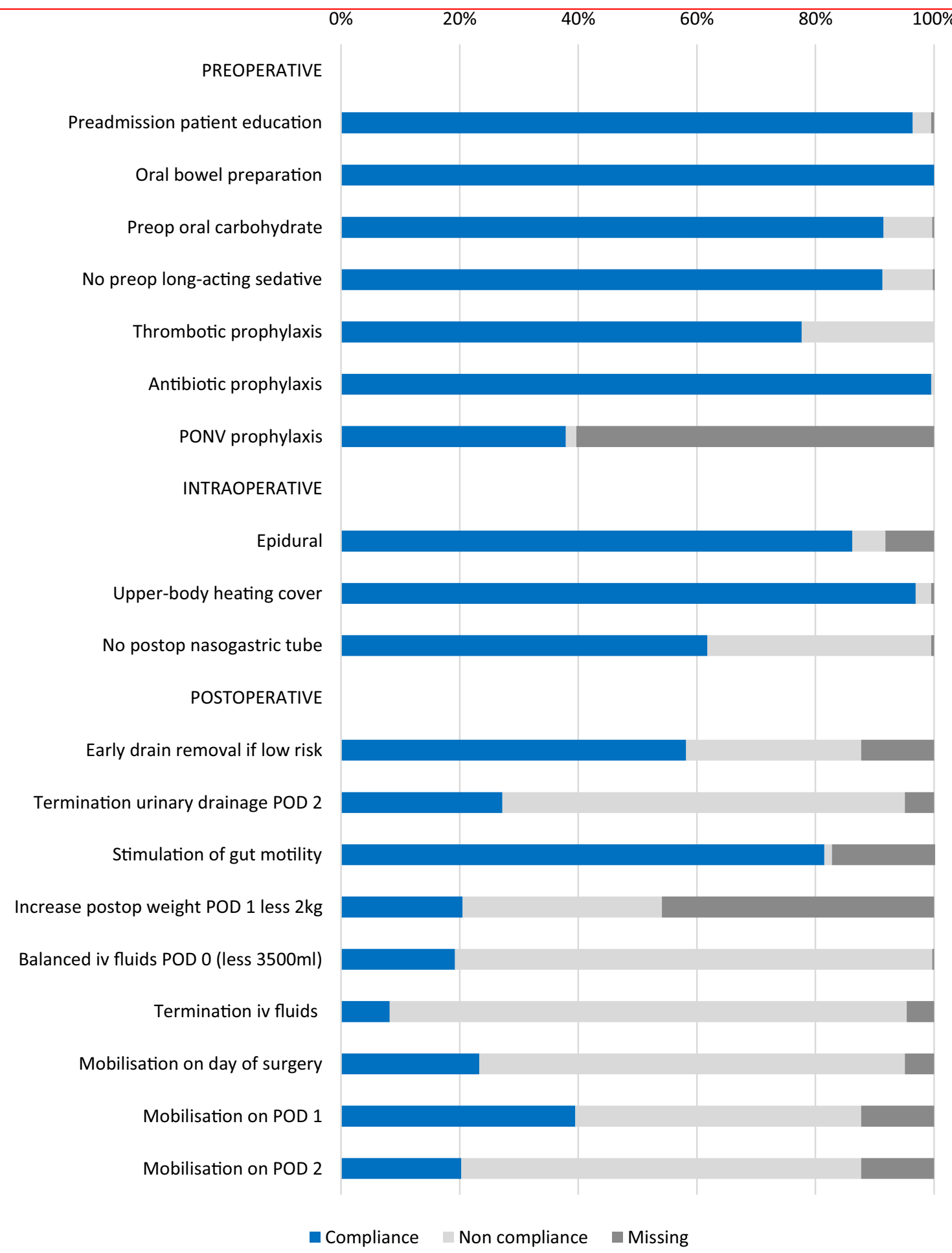

PONV, postoperative nausea and vomiting; POD, postoperative day

Fig. 1 Compliance by ERAS element in patients undergoing pancreatoduodenectomy. ERAS protocol compliance by element in the preoperative, intraoperative and postoperative phases for patients undergoing elective pancreatoduodenectomy. $P O N V$ postoperative nausea and vomiting, $P O D$ postoperative day 
Table 3 Postoperative outcomes of patients undergoing elective pancreatoduodenectomy within an ERAS pathway $(n=390)$

\begin{tabular}{lll}
\hline Category & $N$ & $\%$ \\
\hline Primary length of stay, median (IQR) & $14(9-22)$ & \\
Readmission & 44 & 11.3 \\
Total length of stay, median (IQR) & $15(10-24)$ & \\
Overall complications & 338 & 83.7 \\
Grading of most severe complication & & \\
1-2 (minor) & 180 & 46.2 \\
3-4 (major) & 144 & 36.9 \\
Mortality & 12 & 3.1 \\
Pancreatic surgery specific complications & & \\
$\quad$ Delayed gastric emptying & 130 & 33.3 \\
Clinically relevant pancreatic fistula & 74 & 19.0 \\
$\quad$ Grade B-C) & & 8.7 \\
Post-pancreatectomy hemorrhage & 34 & \\
\hline IQR interquartile range & &
\end{tabular}

$I Q R$ interquartile range

respectively [16]. However, this benchmark was established for low-risk patient excluding those with ASA IV, as opposed to our non-selected study population, which also included high-risk patients.

Several studies [17-21] reported successful outcome following implementation of ERAS for PD. However, only few single center studies reported the obtained compliance to each ERAS element [21-23]. Comparison of compliance between these studies is difficult as the number and definitions of elements included in the enhanced recovery pathway were heterogeneous. Williamsson et al. [21] reported an overall compliance, varying over time from 65 to $72 \%$, similar to the overall compliance observed in this study. The compliance for the pre- and intra-operative periods was consistently high in three previous studies ranging from 84 to $100 \%$ per element [21-23]. Similarly, we observed high compliance in the pre- and intraoperative period with a mean compliance of $93 \%$ and $80 \%$, respectively. Elements with $\geq 90 \%$ compliance were preoperative patient education, avoidance of bowel preparation, carbohydrate loading, avoidance of long-acting sedating medication, antibiotic prophylaxis and use of upper-body forced air heating cover. Only systematic postoperative nausea and vomiting prophylaxis (PONV) was a difficult element in the present study with a compliance of only $38 \%$. Compliance to PONV prophylaxis was previously described in colorectal surgery and lack of prescription by the anesthetist in charge was identified as its reason [24]. If pre- and intra- operative elements are mostly easily achieved, similar conclusion cannot be drawn for the postoperative period. Indeed, the postoperative period contained the most challenging elements to apply in clinical practice. In the present study three main postoperative objective raised concern: fluid balance, urinary drainage removal (average compliance 18.8\%), and adequate postoperative mobilization (average compliance 40.4\%).

Braga et al. reported a compliance of $38 \%$ to $66 \%$ in the postoperative period, with mobilization $(47 \%)$ and intravenous fluid withdrawal (38\%) being the most difficult targets [22]. Williamsson et al. [21] also obtained low compliance in the postoperative period (48-58\%). In the study of Zouros et al. [23] higher compliance was reported in the postoperative period, with $87 \%$ of patients achieving more than $2 \mathrm{~h}$ of mobilization on first postoperative day, but no data were reported on urinary drainage or weight gain. Postoperative elements are the most demanding, as they require supplementary combined effort from caregivers and patients. However, postoperative ERAS elements are essential as they have the greatest impact on optimal recovery as recently shown by a multicenter study in colorectal surgery [25]. In the latter study, the

Table 4 Compliance with ERAS pathway and postoperative outcomes

\begin{tabular}{|c|c|c|c|}
\hline & $\begin{array}{l}\text { Compliance }<70 \% \\
n=305\end{array}$ & $\begin{array}{l}\text { Compliance } \geq 70 \% \\
n=85\end{array}$ & $p$ value \\
\hline Primary length of stay, median (IQR) & $15(10-23)$ & $11(7-16)$ & $<0.001$ \\
\hline Total length of stay, median (IQR) & $15(11-25)$ & $12(7-19)$ & $<0.001$ \\
\hline Overall complications, $n(\%)$ & $271(88.9)$ & $67(78.8)$ & 0.029 \\
\hline Major complications (3-4), $n(\%)$ & $132(43.6)$ & $24(28.2)$ & 0.012 \\
\hline Clinically relevant pancreatic fistula (Grade B-C), $n(\%)$ & $63(20.7)$ & $11(12.9)$ & 0.119 \\
\hline Delayed gastric emptying, $n(\%)$ & $103(34.0)$ & $27(31.8)$ & 0.795 \\
\hline Post-pancreatectomy hemorrhage, $n(\%)$ & $28(9.2)$ & $6(7.1)$ & 0.666 \\
\hline Respiratory complications, $n(\%)$ & $96(31.6)$ & $16(18.8)$ & 0.022 \\
\hline Infectious complications, $n(\%)$ & $118(38.8)$ & $22(25.9)$ & 0.030 \\
\hline Cardiovascular complications, $n(\%)$ & $63(20.7)$ & $13(15.3)$ & 0.284 \\
\hline
\end{tabular}

$I Q R$ interquartile range 
Table 5 Predictive factors for overall complications at 30 postoperative days

\begin{tabular}{|c|c|c|c|c|c|c|}
\hline & \multicolumn{3}{|c|}{ Univariable analysis } & \multicolumn{3}{|c|}{ Multivariable analysis } \\
\hline & OR & $95 \% \mathrm{CI}$ & $p$ value & Adjusted OR & $95 \% \mathrm{CI}$ & $p$ value \\
\hline Age $>70$ years & 0.89 & $0.49-1.63$ & 0.710 & & & \\
\hline Gender (female) & 0.92 & $0.51-1.65$ & 0.780 & & & \\
\hline $\mathrm{BMI}>30 \mathrm{~kg} / \mathrm{m}^{2}$ & 2.54 & $0.88-7.31$ & 0.075 & 1.45 & $0.47-4.50$ & 0.517 \\
\hline ASA III/IV & 2.71 & $1.44-5.08$ & 0.001 & 1.72 & $0.80-3.70$ & 0.169 \\
\hline WHO performance $>0$ & 1.65 & $0.90-3.03$ & 0.104 & & & \\
\hline Length surgery $>300 \mathrm{~min}$ & 1.69 & $0.94-3.05$ & 0.077 & 0.90 & $0.45-1.81$ & 0.766 \\
\hline Malignant tumor & 0.48 & $0.17-1.39$ & 0.166 & & & \\
\hline Patient education & 0.59 & $0.08-4.69$ & 0.616 & & & \\
\hline Thrombotic prophylaxis & 2.79 & $1.51-5.17$ & 0.001 & 1.17 & $0.50-2.75$ & 0.718 \\
\hline No postop nasogastric tube & 0.31 & $0.15-0.66$ & 0.001 & 0.31 & $0.14-0.97$ & 0.043 \\
\hline Early drain removal if low risk & 1.03 & $0.49-2.16$ & 0.930 & & & \\
\hline Termination urinary drainage POD 2 & 1.40 & $0.69-2.86$ & 0.353 & & & \\
\hline Laxatives & 1.00 & $0.46-2.17$ & 0.995 & & & \\
\hline Increased weight POD $1<2 \mathrm{~kg}$ & 0.73 & $0.32-1.66$ & 0.454 & & & \\
\hline Balanced iv fluids POD 0 & 0.58 & $0.30-1.14$ & 0.113 & & & \\
\hline Mobilization on POD 0 & 0.31 & $0.16-0.58$ & $<0.001$ & 0.28 & $0.14-0.97$ & 0.043 \\
\hline Mobilization on POD 1 & 0.72 & $0.39-1.34$ & 0.295 & & & \\
\hline Mobilization on POD 2 & 0.49 & $0.25-0.95$ & 0.033 & 0.45 & $0.22-0.93$ & 0.001 \\
\hline
\end{tabular}

OR odds ratio, BMI body mass index, ASA American Society of Anesthesiologists, WHO World Health Organization, $P O D$ postoperative day

Table 6 Predictive factors for major complications at 30 postoperative days

\begin{tabular}{|c|c|c|c|c|c|c|}
\hline & \multicolumn{3}{|c|}{ Univariable analysis } & \multicolumn{3}{|c|}{ Multivariable analysis } \\
\hline & OR & $95 \% \mathrm{CI}$ & $p$ value & Adjusted OR & $95 \% \mathrm{CI}$ & $p$ value \\
\hline Age $>70$ years & 1.16 & $0.76-1.76$ & 0.501 & & & \\
\hline Gender (female) & 0.55 & $0.36-0.83$ & 0.004 & 0.53 & $0.33-0.85$ & 0.009 \\
\hline $\mathrm{BMI}>30 \mathrm{~kg} / \mathrm{m}^{2}$ & 1.23 & $0.71-2.12$ & 0.453 & & & \\
\hline ASA III/IV & 1.73 & $1.14-2.61$ & 0.009 & 1.47 & $0.87-2.48$ & 0.150 \\
\hline WHO performance $>0$ & 2.17 & $1.35-3.51$ & 0.001 & 2.43 & $1.35-4.36$ & 0.003 \\
\hline Length surgery $>300 \mathrm{~min}$ & 0.88 & $0.58-1.35$ & 0.563 & & & \\
\hline Malignant tumor & 0.85 & $0.48-1.52$ & 0.587 & & & \\
\hline Patient education & 0.47 & $0.15-1.50$ & 0.192 & & & \\
\hline Thrombotic prophylaxis & 1.83 & $1.09-3.07$ & 0.022 & 1.05 & $0.48-2.31$ & 0.905 \\
\hline No postop nasogastric tube & 0.64 & $0.42-0.97$ & 0.033 & 0.69 & $0.39-01.22$ & 0.201 \\
\hline Early drain removal if low risk & 1.64 & $1.03-2.62$ & 0.037 & 1.50 & $0.91-2.46$ & 0.114 \\
\hline Termination urinary drainage POD 2 & 0.82 & $0.51-1.30$ & 0.387 & & & \\
\hline Laxatives & 0.69 & $0.41-1.18$ & 0.174 & & & \\
\hline Increased weight POD $1<2 \mathrm{~kg}$ & 0.69 & $0.39-1.23$ & 0.207 & & & \\
\hline Balanced iv fluids POD 0 & 0.84 & $0.50-1.42$ & 0.520 & & & \\
\hline Mobilization on POD 0 & 0.38 & $0.22-0.65$ & $<0.001$ & 0.42 & $0.22-0.77$ & 0.005 \\
\hline Mobilization on POD 1 & 0.94 & $0.61-1.46$ & 0.782 & & & \\
\hline Mobilization on POD 2 & 1.12 & $0.67-1.87$ & 0.688 & & & \\
\hline
\end{tabular}

$O R$ odds ratio, BMI body mass index, $A S A$ American Society of Anesthesiologists, WHO World Health Organization, $P O D$ postoperative day 
compliance for postoperative elements was $40.3 \%$ among 2876 patients undergoing colorectal surgery [25].

The relationship between increased ERAS compliance and reduced postoperative complication was clearly established in a large international observational study for colorectal surgery [4]. For PD, previous series with 75 to 160 patients [21,23] also reported that an increasing ERAS compliance was associated with reduced morbidity and shortened length of stay. We observed more favorable postoperative outcome when patient overall compliance was more than $70 \%$, especially for respiratory complications and infectious complications. Of note, only less than a quarter of patients achieved such a good compliance and more efforts are still needed to increase compliance rates as well as to adapt the existing guidelines to the specificity of pancreatic resections.

Surprisingly, patients' characteristics and surgery related factors did not interfere with overall complication. With a majority of elderly patients, age itself was not a predictive factor for complications. The preoperative performance status, frequently altered in elderly patients, was an independent factor of major complication. A careful screening of the nutritional status and potential prehabilitation program might be of importance in these patients with altered preoperative performance [26]. Female gender was associated with a reduced risk of major complications, which is consistent with previous studies, which identified male gender as a predictor of complications as well as clinically relevant pancreatic fistula $[27,28]$.

Considering the impact of each individual ERAS element, only postoperative parameters were independent predictors of complications. For overall complications, avoidance of nasogastric tube as well as sufficient mobilization on the day of surgery and on postoperative day 2 were associated with reduced overall complications. Mobilization on postoperative day 2 was the only independent factor for reduced major complication. To our knowledge, no preexisting study analyzed the impact of each individual ERAS element in PD. Despite these postoperative elements are independently correlated with favorable outcome, they are partially modifiable factors, as postoperative complication itself can impinge proper postoperative mobilization. Therefore, mobilization elements can reflect the occurrence of complication. However, patient not able to be mobilized on the day of surgery definitely need more attention since they are more prone to develop complications.

One of the limitations of this study is the missing data, especially for compliance elements, which reached up to ten percent. Data were provided by four tertiary referral centers in four different countries. Since each participating center included in the database the patients, a potential limitation is selection bias. However, each center entered consecutive patients prospectively. A further limitation is that no details on compliance for liquid or solid feeding could be provided due to lack of consensus in the current guidelines and no specific data were available on postoperative diet. However, early diet at will was encouraged.

This large international cohort study suggested that high compliance to ERAS protocol is difficult to achieve for PD, particularly in the postoperative period. When achieved, high compliance was associated with improved outcome.

Acknowledgements The authors would like to thank all members of their respective ERAS team. A special thanks to Valerie Addor, ERAS-dedicated clinical nurse at Lausanne University Hospital.

\section{References}

1. Ljungqvist O, Scott M, Fearon KC (2017) Enhanced recovery after surgery: a review. JAMA Surg 152:292-298

2. Lassen K, Coolsen MM, Slim K et al (2012) Guidelines for perioperative care for pancreaticoduodenectomy: enhanced recovery after surgery (ERAS(R)) society recommendations. Clin Nutr 31:817-830

3. McLeod RS, Aarts MA, Chung F et al (2015) Development of an enhanced recovery after surgery guideline and implementation strategy based on the knowledge-to-action cycle. Ann Surg 262:1016-1025

4. Group EC (2015) The impact of enhanced recovery protocol compliance on elective colorectal cancer resection: results from an international registry. Ann Surg 261:1153-1159

5. von Elm E, Altman DG, Egger M et al (2008) The strengthening the reporting of observational studies in epidemiology (STROBE) statement: guidelines for reporting observational studies. J Clin Epidemiol 61:344-349

6. Elias KM, Stone AB, McGinigle K et al (2019) The reporting on eras compliance, outcomes, and elements research (RECOvER) checklist: a joint statement by the $\operatorname{ERAS}((\mathrm{R}))$ and $\operatorname{ERAS}((\mathrm{R}))$ USA societies. World J Surg 43:1-8

7. Dindo D, Demartines N, Clavien PA (2004) Classification of surgical complications: a new proposal with evaluation in a cohort of 6336 patients and results of a survey. Ann Surg 240:205-213

8. Bassi C, Dervenis C, Butturini G et al (2005) Postoperative pancreatic fistula: an international study group (ISGPF) definition. Surgery 138:8-13

9. Wente MN, Bassi C, Dervenis C et al (2007) Delayed gastric emptying (DGE) after pancreatic surgery: a suggested definition by the International Study Group of Pancreatic Surgery (ISGPS). Surgery 142:761-768

10. Wente MN, Veit JA, Bassi C et al (2007) Postpancreatectomy hemorrhage (PPH): an International Study Group of Pancreatic Surgery (ISGPS) definition. Surgery 142:20-25

11. Bassi C, Marchegiani G, Dervenis C et al (2017) The 2016 update of the International Study Group (ISGPS) definition and grading of postoperative pancreatic fistula: 11 years after. Surgery 161:584-591

12. Owens WD, Felts JA, Spitznagel EL Jr (1978) ASA physical status classifications: a study of consistency of ratings. Anesthesiology 49:239-243

13. Oken MM, Creech RH, Tormey DC et al (1982) Toxicity and response criteria of the Eastern Cooperative Oncology Group. Am J Clin Oncol 5:649-655 
14. Gustafsson UO, Hausel J, Thorell A et al (2011) Adherence to the enhanced recovery after surgery protocol and outcomes after colorectal cancer surgery. Arch Surg 146:571-577

15. Jurt J, Slieker J, Frauche P et al (2017) Enhanced recovery after surgery: can we rely on the key factors or do we need the Bel ensemble? World J Surg 41:2464-2470

16. Sanchez-Velazquez P, Muller X, Malleo G et al (2019) Benchmarks in pancreatic surgery: a novel tool for unbiased outcome comparisons. Ann Surg 270:211-218

17. Coolsen MM, van Dam RM, Chigharoe A et al (2014) Improving outcome after pancreaticoduodenectomy: experiences with implementing an enhanced recovery after surgery (ERAS) program. Dig Surg 31:177-184

18. Dai J, Jiang Y, Fu D (2017) Reducing postoperative complications and improving clinical outcome: enhanced recovery after surgery in pancreaticoduodenectomy-a retrospective cohort study. Int J Surg 39:176-181

19. Joliat GR, Labgaa I, Petermann D et al (2015) Cost-benefit analysis of an enhanced recovery protocol for pancreaticoduodenectomy. Br J Surg 102:1676-1683

20. Robertson N, Gallacher PJ, Peel N et al (2012) Implementation of an enhanced recovery programme following pancreaticoduodenectomy. HPB (Oxford) 14:700-708

21. Williamsson C, Karlsson T, Westrin M et al (2019) Sustainability of an enhanced recovery program for pancreaticoduodenectomy with pancreaticogastrostomy. Scand J Surg 108:17-22

22. Braga M, Pecorelli N, Ariotti R et al (2014) Enhanced recovery after surgery pathway in patients undergoing pancreaticoduodenectomy. World J Surg 38:2960-2966
23. Zouros E, Liakakos T, Machairas A et al (2016) Improvement of gastric emptying by enhanced recovery after pancreaticoduodenectomy. Hepatobiliary Pancreat Dis Int 15:198-208

24. Roulin D, Muradbegovic M, Addor V et al (2017) Enhanced recovery after elective colorectal surgery-reasons for noncompliance with the protocol. Dig Surg 34:220-226

25. Aarts MA, Rotstein OD, Pearsall EA et al (2018) Postoperative ERAS interventions have the greatest impact on optimal recovery: experience with implementation of ERAS across multiple hospitals. Ann Surg 267:992-997

26. Kitahata Y, Hirono S, Kawai M et al (2018) Intensive perioperative rehabilitation improves surgical outcomes after pancreaticoduodenectomy. Langenbecks Arch Surg 403:711-718

27. Aoki S, Miyata H, Konno H et al (2017) Risk factors of serious postoperative complications after pancreaticoduodenectomy and risk calculators for predicting postoperative complications: a nationwide study of 17,564 patients in Japan. J Hepatobiliary Pancreat Sci 24:243-251

28. Partelli S, Tamburrino D, Cherif R et al (2019) Risk and predictors of postoperative morbidity and mortality after pancreaticoduodenectomy for pancreatic neuroendocrine neoplasms: a comparative study with pancreatic ductal adenocarcinoma. Pancreas 48:504-509

Publisher's Note Springer Nature remains neutral with regard to jurisdictional claims in published maps and institutional affiliations. 